\title{
Mind the Gaps: Facilitators and Barriers to Behavioral Change Related to Infant Mortality Rate
}

Jessica Chiang ${ }^{1}$, Sarah Roth ${ }^{2}$, Wilma Griffin², Paige DeChant ${ }^{2}$, and Debra Litzelman ${ }^{1,2,3,4}$

${ }^{1}$ Indiana University School of Medicine, ${ }^{2}$ Regenstrief Institute, ${ }^{3}$ Indiana University Center for Global Health, ${ }^{4}$ Indiana University Health

\section{Background and Hypothesis:}

Marion County suffers from stark racial disparities in infant mortality rate (IMR) with black babies experiencing an IMR of 11.6 and white babies 4.5, to culminate at a state average of 7.3. Since 2016, WeCare Indiana has worked to reduce the IMR in Central Indiana by connecting pregnant, post-partum, and childbearing age women with Community Health Workers (CHW) and community organizations that help with areas including but not limited to baby supplies, housing, and food insecurity.

The goal of this research was to identify factors that promote changes in behaviors in WeCare clients as well as barriers to behavior modification in relation to the five pillars of the WeCare program: food insecurity, mental health, smoking, breastfeeding, and safe sleep.

\section{Experimental Design or Project Methods:}

Qualitative analysis of unstructured CHW follow-up notes from 2016 to 2018 of 1775 WeCare clients from mostly 13 highest risk zip codes for IM in Central Indiana was completed using keyword searches and clustering narrative entries into themes associated with the apriori areas of behavioral change.

\section{Results:}

Several key influencers of behavioral change were identified: (1) CHW dissemination of eligibility information about WIC, (2) CHW referral of clients to behavioral health resources, and (3) Pack n Play resources bundled with Safe Sleep class. Persistent barriers to positive behavioral change included: (1) lack of transportation to food resources, (2) unmanaged stress propagating substance use and smoking, (3) employment interfering with breastfeeding, and (4) lack of Safe Sleep education for all caretakers.

\section{Conclusion and Potential Impact:}

Next steps include sharing qualitative findings with $\mathrm{CHW}$ to reinforce positive behavioral change as well as investigating solutions for the barriers that prevent change within the five pillars of WeCare Indiana. Data from this study will be used to help interpret the calculated behavioral change scores relative to baseline measures that were found from quantitative analysis. 